\title{
About the joint spectrum of vector-valued functions
}

\author{
MART ABEL
}

Abstract. We generalize the results of Abtahi and Farhangi about the joint spectrum and $A$-valued spectrum of a vector-valued map from the class of unital commutative semisimple Banach algebras to the case of unital topological algebras.

\section{Introduction}

For any two sets $S$ and $T$, let $T^{S}$ denote the collection of all maps $f: S \rightarrow T$. If $S$ and $T$ are linear spaces, then $L(S, T)$ denotes the collection of all linear maps $f: S \rightarrow T$.

Let $\mathbb{K}$ denote one of the fields $\mathbb{R}$ of real numbers or $\mathbb{C}$ of complex numbers. Let $X$ be a topological space, and let $A$ be a unital topological algebra over $\mathbb{K}$ with unit $e_{A}$. By $C(X, A)$ we denote the collection of all continuous maps $f: X \rightarrow A$ and by $\mathfrak{M}(A)$ the set of all characters of $A$, i.e., the set of all algebra homomorphisms $\phi: A \rightarrow \mathbb{K}$. Recall that the map $\phi: A \rightarrow \mathbb{K}$ is an algebra homomorphism if $\phi(\lambda a+\mu b)=\lambda \phi(a)+\mu \phi(b)$ and $\phi(a b)=\phi(a) \phi(b)$ for every $a, b \in A, \lambda, \mu \in \mathbb{K}$, and $\phi\left(e_{A}\right)=1$. Notice that the set $\mathfrak{M}(A)$ may be empty for some unital topological algebras, because the map $\phi_{0}: A \rightarrow \mathbb{K}$, which is defined by $\phi_{0}(a)=0$ for every $a \in A$, does not fulfill the condition $\phi_{0}\left(e_{A}\right)=1$ of an algebra homomorphism.

For every pair of maps $\lambda \in \mathbb{K}^{X}$ and $f \in A^{X}$, put

$$
\begin{aligned}
A_{\lambda, f}=\{ & \left\{\sum_{i=1}^{n} a_{i}\left(\lambda\left(x_{i}\right) e_{A}-f\left(x_{i}\right)\right) b_{i}: \quad n \in \mathbb{N},\right. \\
& \left.a_{1}, \ldots, a_{n}, b_{1}, \ldots, b_{n} \in A, \quad x_{1}, \ldots, x_{n} \in X\right\} .
\end{aligned}
$$

Received November 3, 2019.

2010 Mathematics Subject Classification. Primary 46H20; Secondary 46L52, 46H99, 47A10.

Key words and phrases. Unital topological algebra, joint spectrum, $A$-valued spectrum, equicontinuity, uniform closedness, uniform boundedness.

https://doi.org/10.12697/ACUTM.2020.24.10 
Notice that our definition of the general element of the set $A_{\lambda, f}$ is similar to the element used in the definition of the joint spectrum in [2]. As we did not assume the commutativity of our algebra, we had to add here also the elements $b_{1}, \ldots, b_{n}$.

The joint spectrum or vector-valued spectrum of $f \in A^{X}$ is defined in [2]. There it is denoted by $E-S P(f)$. We will denote it by $\operatorname{jsp}(f)$ and define as follows:

$$
\operatorname{jsp}(f)=\left\{\lambda: X \rightarrow \mathbb{K}: e_{A} \notin A_{\lambda, f}\right\} .
$$

By this definition, it is obvious that a map $\lambda \in \mathbb{K}^{X}$ satisfies the condition $\lambda \notin \mathrm{j} \operatorname{sp}(f)$ if and only if there exist $n \in \mathbb{N}, a_{1}, \ldots, a_{n}, b_{1}, \ldots, b_{n} \in A$, and $x_{1}, \ldots, x_{n} \in X$ such that

$$
e_{A}=\sum_{i=1}^{n} a_{i}\left(\lambda\left(x_{i}\right) e_{A}-f\left(x_{i}\right)\right) b_{i} .
$$

During the years, there have been several attempts of describing different spectra of elements of algebras. For an overview of the development of different spectra, we recommend to read the paper [2], where the historical background of different notions of spectra is well explained. We also advise to check the Section 6 of [2], if someone wants to see some examples, where some of the results of the present paper hold in special cases.

In [2], several results about the equivalent descriptions and compactness of the $A$-valued spectrum were proved for a unital commutative semisimple Banach algebra $A$. The study of these results and their proofs showed that analogous results (with exception of the compactness) will hold also for unital topological algebras, when one just puts slighter restrictions that are actually used in the proofs. Moreover, in [2], in several places some identifications of different notions were made in order to simplify the writing. In this paper, we decided not to use identifications in order to simplify the understanding of the proofs. Of course, this made some results and proofs longer and more technical, but at least there will not be the possibility of confusion whether the symbol $a$ stands for an element of $A$ or for a map $\delta_{a}: X \rightarrow A$ for which $\delta_{a}(x)=a$ for every $x \in X$. Another thing that we avoided in this paper, is the usage of so called "admissible vector-valued algebras", which are used in [2]. We also dropped the assumption that the maps for which one wants to find a spectrum, have to be continuous, and used subsets $\mathfrak{G}$ of $\mathfrak{M}(A)$ in the results instead of the whole character set $\mathfrak{M}(A)$. This enables us to obtain more general results (which will obviously hold also, when one takes $\mathfrak{G}=\mathfrak{M}(A))$.

\section{Results about joint spectrum}

We start the paper by generalizing [2, Theorem 3.1]. 
Lemma 1. Let $X$ be a topological space and let $A$ be a unital topological algebra. Assume that $\mathfrak{G} \subseteq \mathfrak{M}(A)$ and $f \in A^{X}$. Then

(i) for every $\phi \in \mathfrak{G}$, we have $\phi \circ f \in \operatorname{jsp}(f)$;

(ii) if for every proper two-sided ideal I of A there exists $\phi \in \mathfrak{G}$ such that $I \subseteq \operatorname{ker}(\phi)$, then $\operatorname{jsp}(f)=\{\phi \circ f: \phi \in \mathfrak{G}\}$ and $\mathfrak{G}=\mathfrak{M}(A)$.

Proof. (i) If $\mathfrak{G}=\emptyset$, then there is nothing to prove.

Otherwise, take any $\phi \in \mathfrak{G}$ and suppose that $\phi \circ f \notin \mathrm{jsp}(f)$. Then there exist $n \in \mathbb{N}, a_{1}, \ldots, a_{n}, b_{1}, \ldots, b_{n} \in A$, and $x_{1}, \ldots, x_{n} \in X$ such that

$$
e_{A}=\sum_{i=1}^{n} a_{i}\left((\phi \circ f)\left(x_{i}\right) e_{A}-f\left(x_{i}\right)\right) b_{i} .
$$

Applying $\phi$ to both sides of this equality, we obtain

$$
1=\phi\left(e_{A}\right)=\sum_{i=1}^{n} \phi\left(a_{i}\right)\left(\phi\left(f\left(x_{i}\right)\right)-\phi\left(f\left(x_{i}\right)\right)\right) \phi\left(b_{i}\right)=0,
$$

which is a contradiction. Hence, $\phi \circ f \in \operatorname{jsp}(f)$.

(ii) Take any $\lambda \in \operatorname{jsp}(f)$ and consider the smallest two-sided ideal $I$ of $A$ generated by the set $\left\{\lambda(x) e_{A}-f(x): x \in X\right\}$. Then $I=A_{\lambda, f}$, which means that $e_{A} \notin I$ and $I$ is a proper two-sided ideal of $A$.

By the assumption, there exists $\phi \in \mathfrak{G}$ such that $I \subseteq \operatorname{ker}(\phi)$. Thus, $(\lambda-(\phi \circ f))(x)=\phi\left(\lambda(x) e_{A}-f(x)\right)=0$ for every $x \in X$. Therefore, $\lambda=\phi \circ f$ and $\operatorname{jsp}(f)=\{\phi \circ f: \phi \in \mathfrak{G}\}$.

Take any $\psi \in \mathfrak{M}(A)$. Then $I=\operatorname{ker}(\psi)$ is a proper two-sided ideal of $A$. Hence, by assumption, there exists $\phi \in \mathfrak{G}$ such that $I \subseteq \operatorname{ker}(\phi)$. Since the kernels of algebra homomorphisms are maximal two-sided ideals, from $\operatorname{ker}(\psi) \subseteq \operatorname{ker}(\phi)$ it follows that $\operatorname{ker}(\psi)=\operatorname{ker}(\phi)$. But different algebra homomorphisms have different kernels. Thus, $\psi=\phi \in \mathfrak{G}$. Since this holds for arbitrary $\psi \in \mathfrak{M}(A)$, we have $\mathfrak{G}=\mathfrak{M}(A)$.

Remark 1. We would like to point out that the assumption (ii) of the following result is automatically fulfilled if $A$ is any commutative GelfandMazur algebra ${ }^{1}$, in which all maximal ideals are closed.

Indeed, if $I$ is any proper ideal of a commutative unital topological algebra $A$, in which all maximal ideals are closed, then there exists a (proper) closed maximal (regular) ideal $M$ of $A$ such that $I \subseteq M$. Let $\kappa_{M}: A \rightarrow A / M$ be the quotient map. If $A$ is also a Gelfand-Mazur algebra, then there exists a topological isomorphism $j: A / M \rightarrow \mathbb{K}$. Taking $\phi=j \circ \kappa_{M}$, it is easy to see that $\phi \in \mathfrak{M}(A)$ and $M=\operatorname{ker}(\phi)$.

\footnotetext{
${ }^{1}$ By a Gelfand-Mazur algebra we mean a topological algebra, for which the quotient algebra $A / M$ is topologically isomorphic to $\mathbb{K}$ for any (proper) closed regular two-sided ideal $M$ of $A$, which is maximal as a left ideal or as a right ideal.
} 
As every Banach algebra is a Gelfand-Mazur algebra, Theorem 3.1 of [2] follows from the previous lemma as a corollary with $\mathfrak{G}=\mathfrak{M}(A)$.

Let $Y$ be a topological space and let $Z$ be a topological linear space. A set $\mathfrak{F} \subseteq Z^{Y}$ is called equicontinuous, if, for every $y \in Y$ and every neighbourhood $O$ of zero in $Z$, there exists a neighbourhood $U$ of $y \in Y$ such that $f(x)-$ $f(y) \in O$ for every $x \in U$ and every $f \in \mathfrak{F}$.

Remark 2. Notice that if $Y$ is a topological linear space and every $f \in \mathfrak{F}$ is a linear map, then $\mathfrak{F}$ becomes equicontinuous if and only if, for every neighbourhood $O$ of zero in $Z$, there exists a balanced neighbourhood $V$ of zero in $Y$ such that $f(V) \subseteq O$ for every $f \in \mathfrak{F}$.

Indeed, if $\mathfrak{F}$ is equicontinuous, taking $y=\theta_{Y}$ in the definition of equicontinuity, we find a balanced neighbourhood $V \subseteq U$ of zero in $Y$ such that $f(V) \subseteq O$ for every $f \in \mathfrak{F}$.

Now, suppose that, for every neighbourhood $O$ of zero in $Z$, there exists a balanced neighbourhood $V$ of zero in $Y$ such that $f(V) \subseteq O$ for every $f \in \mathfrak{F}$. Fix any $y \in Y$ and choose $U=y+V=\{y+v: v \in V\}$. Then $U$ is a neighbourhood of $y$ in $Y$ and every $x \in U$ can be written as $x=y+v_{x}$ for some $v_{x} \in V$. Now, for every $f \in \mathfrak{F}$, we obtain, by linearity of $f$, that

$f(x)-f(y)=f\left(y+v_{x}\right)-f(y)=f(y)+f\left(v_{x}\right)-f(y)=f\left(v_{x}\right) \in f(V) \subset O$ for every $f \in \mathfrak{F}$. Hence, $\mathfrak{F}$ is equicontinuous.

Remark 3. Notice that in the definition of equicontinuity we can also use the condition $f(y)-f(x) \in O$ instead of $f(x)-f(y) \in O$ because we can find inside $O$ a balanced neighbourhood $O^{\prime}$ for which $f(x)-f(y) \in O^{\prime}$ if and only if $f(y)-f(x) \in O^{\prime}$.

Let $X$ be a topological space, let $A, B$ be two topological linear spaces, and let $\mathfrak{F}, \mathfrak{G}$ be some subsets of $A^{X}$ and $L(A, B)$, respectively. Lemma 3.3 of [2] claims that a family $\{\phi \circ f: \phi \in \mathfrak{G}, f \in \mathfrak{F}\} \subseteq B^{X}$ is equicontinuous if $A$ is a unital commutative semisimple Banach algebra, $B=\mathbb{C}, \mathfrak{F} \subseteq C(X, E)$, and $\mathfrak{G}=\mathfrak{M}(A)$. In what follows, we show in the next six results the equicontinuity of similar types of families, using different extra conditions on a (not necessarily unital) topological algebra or some sets defined by that algebra. Some of those extra conditions are automatically fulfilled in case of a unital commutative semisimple Banach algebra. We start with the generalization, where $X$ is a topological space, $A, B$ are topological linear spaces, and the sets $\mathfrak{F} \subseteq A^{X}$ and $\mathfrak{G} \subseteq L(A, B)$ are both equicontinuous.

Lemma 2. Let $X$ be a topological space and let $A, B$ be two topological linear spaces. Suppose that the sets $\mathfrak{F} \subseteq A^{X}$ and $\mathfrak{G} \subseteq L(A, B)$ are equicontinuous. Then the set $\mathfrak{H}=\{\phi \circ f: \phi \in \mathfrak{G}, f \in \mathfrak{F}\}$ is also equicontinuous.

Proof. Fix any $x \in X$ and any neighbourhood $O$ of zero in $B$. As the set $\mathfrak{G}$ is equicontinuous, then, by Remark 2 , there exists a balanced neighbourhood 
$V$ of zero in $A$ such that $\phi(V) \subseteq O$ for every $\phi \in \mathfrak{G}$. Now, fix any $a \in A$ and consider the set $W_{a}=a+V=\{a+v: v \in V\}$. Notice that, for any $b \in W_{a}$, there exists $v_{b} \in V$ such that $b=a+v_{b}$. But then, by the linearity of $\phi$, we obtain

$$
\phi(b)-\phi(a)=\phi\left(a+v_{b}\right)-\phi(a)=\phi(a)+\phi\left(v_{b}\right)-\phi(a)=\phi\left(v_{b}\right) \in \phi(V) \subseteq O,
$$

for every $b \in W_{a}$ and every $\phi \in \mathfrak{G}$.

Since the set $\mathfrak{F}$ is equicontinuous, there exists a neighbourhood $U$ of $x$ in $X$ such that $f(x)-f(y) \in V$ for every $y \in U$ and every $f \in \mathfrak{F}$. Fix any $f \in \mathfrak{F}$. Then $f(y) \in f(x)+V=W_{f(x)}$. But now

$$
(\phi \circ f)(x)-(\phi \circ f)(y)=\phi(f(x))-\phi(f(y)) \subseteq O
$$

for every $y \in U$ and every $\phi \in \mathfrak{G}$. As this holds for every $f \in \mathfrak{F}$, the set $\mathfrak{H}$ is equicontinuous.

Corollary 1. Let $X$ be a topological space and let $A$ be a topological algebra. Suppose that the sets $\mathfrak{F} \subseteq A^{X}$ and $\mathfrak{G} \subseteq \mathfrak{M}(A)$ are equicontinuous. Then the set $\mathfrak{H}=\{\phi \circ f: \phi \in \mathfrak{G}, f \in \mathfrak{F}\}$ is also equicontinuous.

Proof. The result follows, assuming that $A$ is a topological algebra and $B=\mathbb{K}$ in Lemma 2 .

In the next two results we consider the case, where the set $\mathfrak{F}$ is a singleton, consisting of a continuous map, i.e., $\mathfrak{F}=\{f\}$, where $f$ is continuous.

Lemma 3. Let $X$ be a topological space, let $A, B$ be two topological linear spaces and let $f \in C(X, A)$. If the set $\mathfrak{G} \subseteq B^{A}$ is equicontinuous, then the set $\mathfrak{H}=\{\phi \circ f: \phi \in \mathfrak{G}\}$ is also equicontinuous.

Proof. Notice that the set $\mathfrak{F}=\{f\}$ is an equicontinuous subset of $A^{X}$. Hence, the claim follows from Lemma 2, by taking $\mathfrak{F}=\{f\}$.

Corollary 2. Let $X$ be a topological space, let $A$ be a topological algebra, and let $f \in C(X, A)$. If the set $\mathfrak{G} \subseteq \mathbb{K}^{A}$ is equicontinuous, then the set $\mathfrak{H}=\{\phi \circ f: \phi \in \mathfrak{G}\}$ is also equicontinuous.

Proof. The result follows, assuming that $A$ is a topological algebra and $B=\mathbb{K}$ in Lemma 3 .

Finally, we consider the case when $\mathfrak{F}$ is not assumed to be equicontinuous, but some finiteness and boundedness conditions are put on $\mathfrak{F}$ instead.

Lemma 4. Let $X$ be a topological space, let $A, B$ be two topological linear spaces, and let $\mathfrak{F} \subseteq A^{X}$. Suppose that the set $\mathfrak{G} \subseteq B^{A}$ is equicontinuous and, for every $x \in X$, there exists $n_{x} \in \mathbb{Z}^{+}$such that $\{f(x): f \in \mathfrak{F}\}=$ $\left\{a_{1}, \ldots, a_{n_{x}}\right\}$ and the sets $\mathfrak{F}_{i}=\left\{f \in \mathfrak{F}: f(x)=a_{i}\right\}$ are equicontinuous for every $i \in\left\{1, \ldots, n_{x}\right\}$. Then the set $\mathfrak{H}=\{\phi \circ f: \phi \in \mathfrak{G}, f \in \mathfrak{F}\}$ is also equicontinuous. 
Proof. Fix any $x \in X$ and any neighbourhood $O$ of zero in $B$. Since the set $\mathfrak{G}$ is equicontinuous, for every fixed $a \in A$, there exists a neighbourhood $V_{a}$ of $a$ in $A$ such that $\phi(a)-\phi(b) \in O$ for every $b \in V_{a}$ and every $\phi \in \mathfrak{G}$. Now, there exists a balanced neighbourhood $W_{a}$ of zero in $A$ such that $a+W_{a}=\left\{a+w: w \in W_{a}\right\} \subseteq V_{a}$.

Since the set $\mathfrak{F}_{i}$ is equicontinuous for every $i \in\left\{1, \ldots, n_{x}\right\}$, there are neighbourhoods $U_{1}, \ldots, U_{n_{x}}$ of $x$ in $X$ such that $f(x)-f(y) \in W_{a_{i}}$ for every $y \in U_{i}, f \in \mathfrak{F}_{i}$ and $i \in\left\{1, \ldots, n_{x}\right\}$. Since every $W_{a_{i}}$ is balanced, $f(y) \in f(x)+W_{a_{i}} \subseteq V_{a_{i}}$ for every $y \in U_{i}, f \in \mathfrak{F}_{i}$, and $i \in\left\{1, \ldots, n_{x}\right\}$. Set

$$
U=\bigcap_{i=1}^{n_{x}} U_{i}
$$

Then $U$ is a neighbourhood of $x$ in $X$. But now, $f(y) \in f(x)+W_{f(x)} \subseteq V_{f(x)}$ for every $y \in U$ and every $f \in \mathfrak{F}$. Hence,

$$
(\phi \circ f)(x)-(\phi \circ f)(y)=\phi(f(x))-\phi(f(y)) \in O
$$

for every $y \in U$, every $f \in \mathfrak{F}$ and every $\phi \in \mathfrak{G}$. Therefore, the set $\mathfrak{H}$ is equicontinuous.

Corollary 3. Let $X$ be a topological space, $A$ a topological algebra and $\mathfrak{F} \subseteq A^{X}$. Suppose that the set $\mathfrak{G} \subseteq \mathbb{K}^{A}$ is equicontinuous and, for every $x \in X$, there exists $n_{x} \in \mathbb{Z}^{+}$such that $\{f(x): f \in \mathfrak{F}\}=\left\{a_{1}, \ldots, a_{n_{x}}\right\}$ and the sets $\mathfrak{F}_{i}=\left\{f \in \mathfrak{F}: f(x)=a_{i}\right\}$ are equicontinuous for every $i \in\left\{1, \ldots, n_{x}\right\}$. Then the set $\mathfrak{H}=\{\phi \circ f: \phi \in \mathfrak{G}, f \in \mathfrak{F}\}$ is also equicontinuous.

Proof. The result follows, assuming that $A$ is a topological algebra and $B=\mathbb{K}$ in Lemma 4 .

Again, let $A$ be a topological algebra. Let us remind that, for every $a \in A$, the Gelfand map $\hat{a}: \mathfrak{M}(A) \rightarrow \mathbb{K}$ is defined by $\hat{a}(\phi)=\phi(a)$ for every $\phi \in \mathfrak{M}(A)$.

A Gelfand map $\hat{a}$ is said to be bounded on a set $S \subseteq \mathfrak{M}(A)$ if there exists a positive number $K \in \mathbb{R}$ such that $|\hat{a}(\phi)| \leqslant K$ for every $\phi \in S$.

Let $X$ be a topological space and let $\mathcal{A}$ be a directed set with order relation $\succeq$. We remind that a net $\left(\phi_{\alpha}\right)_{\alpha \in \mathcal{A}}$ of elements of $\mathbb{K}^{X}$ converges uniformly to $\psi \in \mathbb{K}^{X}$ if, for every $\epsilon>0$, there exists $\alpha_{\epsilon} \in \mathcal{A}$ such that

$$
\left|\phi_{\alpha}(x)-\psi(x)\right|<\epsilon
$$

for every $x \in X$ and every $\alpha \in \mathcal{A}$ with $\alpha \succeq \alpha_{\epsilon}$.

A set $S \subseteq \mathbb{K}^{X}$ is uniformly closed if, for every net $\left(\phi_{\alpha}\right)_{\alpha \in \mathcal{A}}$ of elements of $S$ that converges uniformly to $\psi \in \mathbb{K}^{X}$, we have $\psi \in S$.

A collection $\mathfrak{K} \subseteq \mathbb{K}^{X}$ of functions is uniformly bounded if there exists a real number $M>0$ such that $|\kappa(x)| \leqslant M$ for every $\kappa \in \mathfrak{K}$ and every $x \in X$. 
The next proposition gives our version of Theorem 3.4 in [2], We will not obtain the compactness of the joint spectrum, but get the parts that enable to obtain the compactness, when many different assumptions are fulfilled at the same time (as it is the case for the unital Banach algebra).

Proposition 1. Let $X$ be a topological space and let $A$ be a topological algebra. Assume that $f \in A^{X}, \mathfrak{G} \subseteq \mathfrak{M}(A)$, and $A_{f}=\{f(x): x \in X\}$.

(i) If $A$ is unital, $\operatorname{jsp}(f)=\{\phi \circ f: \phi \in \mathfrak{G}\}$, and the Gelfand map $\hat{a}$ is bounded on the set $\mathfrak{G}$ for every $a \in A$, then the set $\operatorname{jsp}(f)$ is uniformly closed in $\mathbb{K}^{X}$.

(ii) If there exists a real number $M>0$ such that $\sup \left\{|\phi(a)|: a \in A_{f}\right\} \leqslant M$ for every $\phi \in \mathfrak{G}$, then the set $\{\phi \circ f: \phi \in \mathfrak{G}\}$ is uniformly bounded.

Proof. (i) Take any $\lambda \in \mathbb{K}^{X} \backslash \operatorname{jsp}(f)=\mathbb{K}^{X} \backslash\{\phi \circ f: \phi \in \mathfrak{G}\}$. Then there exist $n \in \mathbb{N}, a_{1}, \ldots, a_{n}, b_{1}, \ldots, b_{n} \in A$, and $x_{1}, \ldots, x_{n} \in X$ such that

$$
e_{A}=\sum_{i=1}^{n} a_{i}\left(\lambda\left(x_{i}\right) e_{A}-f\left(x_{i}\right)\right) b_{i} .
$$

If $\lambda \in \overline{\{\phi \circ f: \phi \in \mathfrak{G}\}}$, i.e., $\lambda$ belongs to the uniform closure ${ }^{2}$ of the set $\{\phi \circ f: \phi \in \mathfrak{G}\}$, then there exists a net $\left(\phi_{\alpha}\right)_{\alpha \in \mathcal{A}}$ of elements of $\mathfrak{G}$ such that $\left(\phi_{\alpha} \circ f\right)_{\alpha \in \mathcal{A}}$ converges uniformly to $\lambda$. As all the Gelfand maps are bounded on $\mathfrak{G}$, then also the maps $a_{1}, \ldots, a_{n}, b_{1}, \ldots, b_{n}$ are uniformly bounded, which means that there exist positive numbers $K_{1}, \ldots, K_{n}, L_{1}, \ldots, L_{n} \in \mathbb{R}$ with $\left|\phi\left(a_{i}\right)\right|=\left|a_{i}(\phi)\right| \leqslant K_{i}$ and $\left|\phi\left(b_{i}\right)\right|=\left|b_{\hat{i}}(\phi)\right| \leqslant L_{i}$ for each $\phi \in \mathfrak{G}$ and each $i \in\{1, \ldots, n\}$. Let $K=\max \left\{K_{1}, \ldots, K_{n}\right\}$ and $L=\max \left\{L_{1}, \ldots, L_{n}\right\}$. Take $\epsilon=1 /(n K L)$.

As $\left(\phi_{\alpha} \circ f\right)_{\alpha \in \mathcal{A}}$ converges uniformly to $\lambda$, there exists $\alpha_{\epsilon} \in \mathcal{A}$ such that

$$
\left|\lambda(x)-\left(\phi_{\alpha_{\epsilon}} \circ f\right)(x)\right|=\left|\left(\phi_{\alpha_{\epsilon}} \circ f\right)(x)-\lambda(x)\right|<\epsilon
$$

for each $x \in X$. Hence,

$$
\begin{aligned}
1 & =\left|\phi_{\alpha_{\epsilon}}\left(e_{A}\right)\right|=\left|\phi_{\alpha_{\epsilon}}\left(\sum_{i=1}^{n} a_{i}\left(\lambda\left(x_{i}\right) e_{A}-f\left(x_{i}\right)\right) b_{i}\right)\right| \\
& \left.\leq \sum_{i=1}^{n}\left|\phi_{\alpha_{\epsilon}}\left(a_{i}\right)\right| \cdot \mid \lambda\left(x_{i}\right)-\left(\phi_{\alpha_{\epsilon}} \circ f\right)\left(x_{i}\right)\right)|\cdot| \phi_{\alpha_{\epsilon}}\left(b_{i}\right) \mid \\
& <\sum_{i=1}^{n} K \epsilon L=(n K L) \epsilon=1 .
\end{aligned}
$$

But $1<1$ is a contradiction. Thus $\lambda \notin \overline{\{\phi \circ f: \phi \in \mathfrak{G}\}}$, which means that the set $\{\phi \circ f: \phi \in \mathfrak{G}\}$ is uniformly closed in $\mathbb{K}^{X}$.

\footnotetext{
${ }^{2}$ The uniform closure $\bar{S}$ in $T$ of a set $S \subseteq T$ conists of all such elements $t \in T$ for which there exists a net $\left(s_{\alpha}\right)_{\alpha \in \mathcal{A}}$, which converges uniformly to $t$.
} 
(ii) Take any $\phi \in \mathfrak{G}$ and any $x \in X$. Then

$$
|(\phi \circ f)(x)|=|\phi(f(x))| \leqslant \sup \left\{|\phi(a)|: a \in A_{f}\right\} \leqslant M .
$$

Hence, the set $\{\phi \circ f: \phi \in \mathfrak{G}\}$ is uniformly bounded.

Example 1. Let $X$ be a topological space, let $A$ be a unital $Q$-algebra, ${ }^{3}$ and let $\mathfrak{G}=\mathfrak{M}(A)$. It is known (see [3], Proposition 7.1) that $\mathfrak{M}(A)$ is equicontinuous whenever $A$ is a unital $Q$-algebra. Hence, $\mathfrak{M}(A)$ is compact and the Gelfand map $\hat{a}$ is bounded on the set $\mathfrak{G}$ for every $a \in A$. Thus, the conditions of claim (i) of Proposition 1 are fulfilled.

Taking $\mathfrak{G}=C_{b}(X, A)$ (the algebra of all bounded maps $\phi: X \rightarrow A$ ), we obtain that the condition of claim (ii) of Proposition 1 is fulfilled.

As there are many unital $Q$-algebras which are not Banach nor normed algebras, there are many examples of non-normed algebras $A$ and collections $\mathfrak{G} \subseteq \mathfrak{M}(A)$ for which the condition (i) or (ii) of Proposition 1 holds.

Let $X$ be a topological space and let $A$ be a topological algebra. A net $\left(\phi_{\alpha}\right)_{\alpha \in \mathcal{A}}$ of elements of $A^{X}$ converges uniformly to $\psi \in A^{X}$ if for any neighbourhood $O$ of zero in $A$, there exists $\alpha_{O} \in \mathcal{A}$ such that $\phi_{\alpha}(x)-\psi(x) \in O$ for every $x \in X$ and every $\alpha \in \mathcal{A}$ with $\alpha \succeq \alpha_{O}$.

To finish up this section, we present our generalisation of [2, Lemma 3.5].

Proposition 2. Let $X$ be a topological space, let $A$ be a unital topological algebra, let $\mathcal{B}$ be a subalgebra of $A^{X}$, and let $\mathfrak{G} \subseteq \mathfrak{M}(A)$. Suppose that $\left(f_{\alpha}\right)_{\alpha \in \mathcal{A}}$ is a net of elements of $\mathcal{B}$ and $\left(\lambda_{\alpha}\right)_{\alpha \in \mathcal{A}}$ is a net of maps in $\mathbb{K}^{X}$ such that $\lambda_{\alpha} \in\left\{\phi \circ f_{\alpha}: \phi \in \mathfrak{G}\right\}$ for every $\alpha \in \mathcal{A}$. If there are elements $f \in \mathcal{B}$ and $\lambda \in \mathbb{K}^{X}$ such that

(i) the net $\left(f_{\alpha}\right)_{\alpha \in \mathcal{A}}$ converges uniformly to $f$,

(ii) the net $\left(\lambda_{\alpha}\right)_{\alpha \in \mathcal{A}}$ converges uniformly to $\lambda$,

(iii) $\mathfrak{G}$ is equicontinuous,

and

(iv) the Gelfand map $\hat{a}$ is bounded on the set $\mathfrak{G}$ for every $a \in A$, then $\lambda \in \operatorname{jsp}(f)$.

Proof. Suppose that $\lambda \notin \mathrm{j} \operatorname{sp}(f)$. Then there exist $n \in \mathbb{N}, x_{1}, \ldots, x_{n} \in X$, and $a_{1}, \ldots, a_{n}, b_{1}, \ldots, b_{n} \in A$ such that

$$
e_{A}=\sum_{i=1}^{n} a_{i}\left(\lambda\left(x_{i}\right) e_{A}-f\left(x_{i}\right)\right) b_{i} .
$$

By condition (iv) we know that the maps $a_{1}, \ldots, a_{n}, b_{1}, \ldots, b_{n}$ are uniformly bounded on $\mathfrak{G}$, which means that there are positive numbers $K_{1}, \ldots, K_{n}, L_{1}, \ldots, L_{n} \in \mathbb{R}$ with $\left|\phi\left(a_{i}\right)\right|=\left|a_{i}(\phi)\right| \leqslant K_{i}$ and $\left|\phi\left(b_{i}\right)\right|=\left|b_{i}(\phi)\right| \leqslant L_{i}$

\footnotetext{
${ }^{3}$ A unital algebra $A$ is a $Q$-algebra, if the set of all invertible elements of $A$ is open in $A$.
} 
for every $\phi \in \mathfrak{G}$ and every $i \in\{1, \ldots, n\}$. Set $K=\max \left\{K_{1}, \ldots, K_{n}\right\}$ and $L=\max \left\{L_{1}, \ldots, L_{n}\right\}$.

Take $\epsilon=1 /(2 n K L)$. Then the set $S_{\epsilon}=\{\mu \in \mathbb{K}:|\mu|<\epsilon\}$ is a neighbourhood of zero in $\mathbb{K}$.

As $\mathfrak{G}$ is equicontinuous, there exists a neighbourhood $O$ of zero in $A$ such that $\phi(O) \subseteq S_{\epsilon}$ for every $\phi \in \mathfrak{G}$. Since the net $\left(f_{\alpha}\right)_{\alpha \in \mathcal{A}}$ converges uniformly to $f$, there exists $\beta \in \mathcal{A}$ such that $f_{\alpha}(x)-f(x) \in O$ for every $x \in X$ and every $\alpha \in \mathcal{A}$ with $\alpha \succeq \beta$. Because the net $\left(\lambda_{\alpha}\right)_{\alpha \in \mathcal{A}}$ converges uniformly to $\lambda$, there exists $\gamma \in \mathcal{A}$ such that $\lambda_{\alpha}(x)-\lambda(x) \in S_{\epsilon}$ for every $x \in X$ and every $\alpha \in \mathcal{A}$ with $\alpha \succeq \gamma$. As the set $\mathcal{A}$ is directed, there exists $\delta \in \mathcal{A}$ such that $\delta \succeq \alpha$ and $\delta \succeq \bar{\beta}$. Since $\lambda_{\delta} \in\left\{\phi \circ f_{\delta}: \phi \in \mathfrak{G}\right\}$, there exists $\phi_{\delta} \in \mathfrak{G}$ such that $\lambda_{\delta}=\phi_{\delta} \circ f_{\delta}$. Applying $\phi_{\delta}$ to the both sides of the equality (2.1), we obtain that

$$
\begin{aligned}
1= & \left|\phi_{\delta}\left(e_{A}\right)\right|=\left|\phi_{\delta}\left(\sum_{i=1}^{n} a_{i}\left(\lambda\left(x_{i}\right) e_{A}-f\left(x_{i}\right)\right) b_{i}\right)\right| \\
= & \left|\sum_{i=1}^{n} \phi_{\delta}\left(a_{i}\right)\left[\lambda\left(x_{i}\right)-\lambda_{\delta}\left(x_{i}\right)+\left(\phi_{\delta} \circ f_{\delta}\right)\left(x_{i}\right)-\left(\phi_{\delta} \circ f\right)\left(x_{i}\right)\right] \phi_{\delta}\left(b_{i}\right)\right| \\
\leqslant & \sum_{i=1}^{n}\left|\phi_{\delta}\left(a_{i}\right)\right|\left(\left|\lambda\left(x_{i}\right)-\lambda_{\delta}\left(x_{i}\right)\right|+\left|\phi_{\delta}\left(f_{\delta}\left(x_{i}\right)-f\left(x_{i}\right)\right)\right|\right)\left|\phi_{\delta}\left(b_{i}\right)\right| \\
\leqslant & \sum_{i=1}^{n}\left|\phi_{\delta}\left(a_{i}\right)\right|\left|\lambda_{\delta}\left(x_{i}\right)-\lambda\left(x_{i}\right)\right|\left|\phi_{\delta}\left(b_{i}\right)\right| \\
& +\sum_{i=1}^{n}\left|\phi_{\delta}\left(a_{i}\right) \| \phi_{\delta}\left(f_{\delta}\left(x_{i}\right)-f\left(x_{i}\right)\right)\right|\left|\phi_{\delta}\left(b_{i}\right)\right| \\
< & \sum_{i=1}^{n} K \epsilon L+\sum_{i=1}^{n} K \epsilon L=2 n K \epsilon L=1 .
\end{aligned}
$$

But this gives us a contradiction $1<1$. Hence, $\lambda \in \operatorname{jsp}(f)$.

Example 2. Let $X$ be a topological space and let $A$ be a unital $Q$-algebra. Take $\mathfrak{G}=\mathfrak{M}(A)$ and $\mathcal{B}=C_{0}(X, A)$, equipped with the uniform topology. In $C_{0}(X, A)$, there always exists a converging net $\left(f_{\alpha}\right)_{\alpha \in \mathcal{A}}$, which converges also uniformly to some $f \in C_{0}(X, A)$. But then the net $\left(\lambda_{\alpha}\right)_{\alpha \in \mathcal{A}}$ will converge uniformly to $\lambda=\phi \circ f$.

As we mentioned in the Example 1, the map $\hat{a}$ is bounded on the set $\mathfrak{M}(A)$ for every $a \in A$ and $\mathfrak{M}(A)$ is equicontinuous. So the conditions (i), (ii), (iii) and (iv) of Proposition 2 are fulfilled. 


\section{Results about $A$-valued spectrum}

Define a map $\delta_{e_{A}}: X \rightarrow A$ by $\delta_{e_{A}}(x)=e_{A}$ for each $x \in X$. For any $g \in \mathbb{C}^{X} \cup A^{X}$ we will denote by $g \cdot \delta_{e_{A}} \in A^{X}$ a map, which is defined by $\left(g \cdot \delta_{e_{A}}\right)(x)=g(x) \delta_{e_{A}}(x)=g(x) e_{A}$ for every $x \in X$. Then $\delta_{e_{A}}$ will be the unital element of any subalgebra $\mathcal{B}$ of $A^{X}$, which contains $\delta_{e_{A}}$.

In [1] and [2], the original definition of an $A$-character was given. We have modified this definition a bit, resulting in the following definition of an $A$-character.

Definition 1. Let $A$ be a unital topological algebra, let $X$ be a topological space, and let $\mathcal{B}$ be a subalgebra of $A^{X}$, containing the map $\delta_{e_{A}}$. An $A$-character of $\mathcal{B}$ is an algebra homomorphism $\Psi: \mathcal{B} \rightarrow A$ such that $\Psi\left(\delta_{e_{A}}\right)=e_{A}$ and $[\phi(\Psi(f))] e_{A}=\Psi\left[(\phi \circ f) \cdot \delta_{e_{A}}\right]$ for all $f \in \mathcal{B}$ and $\phi \in \mathfrak{M}(A)$. The collection of all $A$-characters of $\mathcal{B}$ will be denoted by $\mathfrak{M}_{A}(\mathcal{B})$.

Let $A$ be a unital topological algebra, let $X$ be a topological space, and let $\mathcal{B}$ be a subalgebra of $A^{X}$, containing the map $\delta_{e_{A}}$. Define a map $\epsilon_{e_{A}}: \mathcal{B} \rightarrow A$ by $\epsilon_{e_{A}}(f)=e_{A}$ for every $f \in \mathcal{B}$. For any map $\psi \in \mathfrak{M}(\mathcal{B})$, define $\psi \cdot \epsilon_{e_{A}} \in A^{\mathcal{B}}$ as follows: $\left(\psi \cdot \epsilon_{e_{A}}\right)(f)=\psi(f) \epsilon_{e_{A}}(f)=\psi(f) e_{A}$ for each $f \in \mathcal{B}$. For every $f \in \mathcal{B}$, define a map $\tilde{f}: \mathfrak{M}(A) \rightarrow \mathcal{B}$ by $\tilde{f}(\phi)=(\phi \circ f) \cdot \delta_{e_{A}}$ for each $\phi \in \mathfrak{M}(A)$.

Definition 2. We say that a character $\psi \in \mathfrak{M}(\mathcal{B})$ lifts to an $A$-character, if there exists an $A$-character $\Psi \in \mathfrak{M}_{A}(\mathcal{B})$ such that $\Psi=\psi \cdot \epsilon_{e_{A}}$.

As in the first part of the present paper, by taking $\mathfrak{M}(A)$ instead of $X$ and $\mathcal{B}$ instead of $A$, we can define the joint spectrum of $\tilde{f}$ as follows:

$$
\operatorname{jsp}(\tilde{f})=\left\{\mu: \mathfrak{M}(A) \rightarrow \mathbb{C}: \delta_{e_{A}} \notin A_{\mu, \tilde{f}}\right\},
$$

where

$$
\begin{aligned}
& A_{\mu, \tilde{f}}=\left\{\sum_{i=1}^{n} g_{i}\left(\mu\left(\phi_{i}\right) \delta_{e_{A}}-\tilde{f}\left(\phi_{i}\right)\right) h_{i}: n \in \mathbb{N},\right. \\
& \left.g_{1}, \ldots, g_{n}, h_{1}, \ldots, h_{n} \in \mathcal{B}, \quad \phi_{1}, \ldots, \phi_{n} \in \mathfrak{M}(A)\right\} .
\end{aligned}
$$

Hence, $\mu \in \operatorname{jsp}(\tilde{f})$ if and only if $\delta_{e_{A}} \notin A_{\mu, \tilde{f}}$.

Definition 3. Let $A$ be a unital topological algebra, let $X$ be a topological space, and let $\mathcal{B}$ be a subalgebra of $A^{X}$, containing the map $\delta_{e_{A}}$. The $A$-valued joint spectrum of a map $f \in \mathcal{B}$ is the set

$$
\operatorname{jsp}_{A}(f)=\{a \in A: \hat{a} \in \mathrm{jsp}(f)\} .
$$

Let us remind that $\mathfrak{M}(A)$ separates the points of $A$ if for every $a_{1}, a_{2} \in A$, with $a_{1} \neq a_{2}$, there exists $\phi \in \mathfrak{M}(A)$ such that $\phi\left(a_{1}\right) \neq \phi\left(a_{2}\right)$.

We finish the paper by generalizing Theorem 4.2 from [2]. 
Theorem 1. Let $A$ be a unital topological algebra, let $X$ be a topological space, and let $\mathcal{B}$ be a subalgebra of $A^{X}$, containing the map $\delta_{e_{A}}$. Fix $f \in \mathcal{B}$.

(i) The following inclusions hold:

$$
f(X) \subseteq\left\{\Psi(f): \Psi \in \mathfrak{M}_{A}(\mathcal{B})\right\} \subseteq \operatorname{jsp}_{A}(f) .
$$

(ii) If $\operatorname{jsp}(\tilde{f})=\{\psi \circ \tilde{f}: \psi \in \mathfrak{M}(\mathcal{B})\}, \mathfrak{M}(A)$ separates the points of $A$ and every character from the set $\mathfrak{M}(\mathcal{B})$ lifts to an $A$-character, then

$$
\operatorname{jsp}_{A}(f)=\left\{\Psi(f): \Psi \in \mathfrak{M}_{A}(\mathcal{B})\right\} .
$$

Proof. (i) For every $x \in X$, there exists a map $\mathcal{E}_{x}: \mathcal{B} \rightarrow A$, defined by $\mathcal{E}_{x}(g)=g(x)$ for each $g \in \mathcal{B}$. Notice that $\mathcal{E}_{x}\left(\delta_{e_{A}}\right)=\delta_{e_{A}}(x)=e_{A}$ and

$$
\begin{aligned}
{\left[\phi\left(\mathcal{E}_{x}(f)\right)\right] e_{A} } & =[\phi(f(x))] \delta_{e_{A}}(x)=[(\phi \circ f)(x)] \delta_{e_{A}}(x) \\
& =\left[(\phi \circ f) \cdot \delta_{e_{A}}\right](x)=\mathcal{E}_{x}\left[(\phi \circ f) \cdot \delta_{e_{A}}\right]
\end{aligned}
$$

for each $x \in X$ and each $\phi \in \mathfrak{M}(A)$. Hence, $\mathcal{E}_{x} \in \mathfrak{M}_{A}(\mathcal{B})$. Thus,

$$
f(X)=\left\{\mathcal{E}_{x}(f): x \in X\right\} \subseteq\left\{\Psi(f): \Psi \in \mathfrak{M}_{A}(\mathcal{B})\right\} .
$$

Take any $\Psi \in \mathfrak{M}_{A}(\mathcal{B})$ and set $a=\Psi(f)$. In order to show that $a \in \operatorname{jsp}_{A}(f)$, it is enough to show that $\hat{a} \in \operatorname{jsp}(\tilde{f})$, i.e., $\delta_{e_{A}} \notin A_{\hat{a}, \tilde{f}}$.

Suppose that $\delta_{e_{A}} \in A_{\hat{a, \tilde{f}}}$. Then there are $n \in \mathbb{N}, g_{1}, \ldots, g_{n}, h_{1}, \ldots, h_{n} \in \mathcal{B}$, and $\phi_{1}, \ldots, \phi_{n} \in \mathfrak{M}(A)$ such that

$$
\delta_{e_{A}}=\sum_{i=1}^{n} g_{i}\left(\hat{a}\left(\phi_{i}\right) \delta_{e_{A}}-\tilde{f}\left(\phi_{i}\right)\right) h_{i} .
$$

As $\Psi \in \mathfrak{M}_{A}(\mathcal{B})$, then $\Psi\left(\delta_{e_{A}}\right)=e_{A}$ and $\left[\phi_{i}(\Psi(f))\right] e_{A}=\Psi\left[\left(\phi_{i} \circ f\right) \cdot \delta_{e_{A}}\right]$ for every $i \in\{1, \ldots, n\}$. On the other hand, since $\tilde{f}\left(\phi_{i}\right)=\left(\phi_{i} \circ f\right) \cdot \delta_{e_{A}}$ and $\Psi(f)=a$,

$$
\begin{aligned}
e_{A} & =\Psi\left(\delta_{e_{A}}\right)=\Psi\left(\sum_{i=1}^{n} g_{i}\left(\hat{a}\left(\phi_{i}\right) \delta_{e_{A}}-\tilde{f}\left(\phi_{i}\right)\right) h_{i}\right) \\
& =\sum_{i=1}^{n} \Psi\left(g_{i}\right)\left(\hat{a}\left(\phi_{i}\right) \Psi\left(\delta_{e_{A}}\right)-\Psi\left(\tilde{f}\left(\phi_{i}\right)\right)\right) \Psi\left(h_{i}\right) \\
& =\sum_{i=1}^{n} \Psi\left(g_{i}\right)\left(\phi_{i}(a) e_{A}-\Psi\left[\left(\phi_{i} \circ f\right) \cdot \delta_{e_{A}}\right]\right) \Psi\left(h_{i}\right) \\
& =\sum_{i=1}^{n} \Psi\left(g_{i}\right)\left(\phi_{i}(a) e_{A}-\left[\phi_{i}(\Psi(f))\right] e_{A}\right) \Psi\left(h_{i}\right) \\
& =\sum_{i=1}^{n} \Psi\left(g_{i}\right)\left(\phi_{i}(a) e_{A}-\phi_{i}(a) e_{A}\right) \Psi\left(h_{i}\right)=\theta_{A} .
\end{aligned}
$$


This is a contradiction, which shows that $\delta_{e_{A}} \notin A_{\hat{a, \tilde{f}}}$. Thus,

$$
\Psi(f)=a \in \operatorname{jsp}_{A}(f) \text { and }\left\{\Psi(f): \Psi \in \mathfrak{M}_{A}(\mathcal{B})\right\} \subseteq \operatorname{jsp}_{A}(f) .
$$

(ii) Take any element $a \in \operatorname{jsp}_{A}(f)$. Then $\hat{a} \in \operatorname{jsp}(\tilde{f})$. Hence, there exists $\psi \in \mathfrak{M}(\mathcal{B})$ such that $\hat{a}=\psi \circ \tilde{f}$. By the assumption, $\psi$ lifts to some $A$-character $\Psi$, i.e, there exists $\Psi \in \mathfrak{M}_{A}(\mathcal{B})$ such that $\Psi=\psi \cdot \epsilon_{e_{A}}$. Now, for every $\phi \in \mathfrak{M}(A)$, we obtain

$$
\begin{aligned}
\phi(a) e_{A} & =\hat{a}(\phi) e_{A}=(\psi \circ \tilde{f})(\phi) e_{A}=\psi(\tilde{f}(\phi)) e_{A} \\
& =\psi\left[(\phi \circ f) \cdot \delta_{e_{A}}\right] e_{A}=\left(\psi \cdot \epsilon_{e_{A}}\right)\left[(\phi \circ f) \cdot \delta_{e_{A}}\right] \\
& =\Psi\left[(\phi \circ f) \cdot \delta_{e_{A}}\right]=[\phi(\Psi(f))] e_{A} .
\end{aligned}
$$

Hence, $\phi(a)=\phi(\Psi(f))$ for every $\phi \in \mathfrak{M}(A)$. As $\mathfrak{M}(A)$ separates the points of $A$, then $a=\Psi(f)$, i.e., $\operatorname{jsp}_{A}(f) \subseteq\left\{\Psi(f): \Psi \in \mathfrak{M}_{A}(\mathcal{B})\right\}$. Combining this result with the result obtained in part (i), we obtain

$$
\operatorname{jsp}_{A}(f)=\left\{\Psi(f): \Psi \in \mathfrak{M}_{A}(\mathcal{B})\right\} .
$$

\section{Acknowledgements}

The research was supported by the Institutional Research Funding IUT20-57 of the Estonian Ministry of Education and Research. The author is grateful to the referee for pointing out that $\mathfrak{G}=\mathfrak{M}(A)$ in part (ii) of Lemma 1.

\section{References}

[1] M. Abtahi, Vector-valued characters on vector-valued function algebras, Banach J. Math. Anal. 10(3) (2016), 608-620.

[2] M. Abtahi and S. Farhangi, Vector-valued spectra of Banach algebra valued continuous functions, Rev. R. Acad. Cienc. Exactas Fís. Nat. Ser. A Mat. RACSAM 112(1) (2018), $103-115$.

[3] A. Mallios, Topological Algebras. Selected Topics. North-Holland Mathematics Studies 124, North-Holland, Amsterdam, 1986.

School of Digital Technologies, Tallinn University, Narva 25, 10120 Tallinn, Estonia; Institute of Mathematics and Statistics, University of Tartu, Narva 18, 51009 TARTU, Estonia

E-mail address: mart.abel@tlu.ee, mart.abel@ut.ee 